doi:10.13108/2013-5-2-18

UDC 517.9

\title{
ON EXISTENCE OF NODAL SOLUTION TO ELLIPTIC EQUATIONS WITH CONVEX-CONCAVE NONLINEARITIES
}

\section{V.E. BOBKOV}

\begin{abstract}
In a bounded connected domain $\Omega \subset \mathbb{R}^{N}, N \geqslant 1$, with a smooth boundary, we consider the Dirichlet boundary value problem for elliptic equation with a convex-concave nonlinearity

$$
\left\{\begin{array}{l}
-\Delta u=\lambda|u|^{q-2} u+|u|^{\gamma-2} u, \quad x \in \Omega \\
\left.u\right|_{\partial \Omega}=0,
\end{array}\right.
$$

where $1<q<2<\gamma<2^{*}$. As a main result, we prove the existence of a nodal solution to this equation on the nonlocal interval $\lambda \in\left(-\infty, \lambda_{0}^{*}\right)$, where $\lambda_{0}^{*}$ is determined by the variational principle of nonlinear spectral analysis via fibering method.
\end{abstract}

Keywords:nodal solution, convex-concave nonlinearity, fibering method

Mathematics Subject Classification: 35D30, 35J25, 35J20, 35J60.

\section{INTRODUCTION}

We consider the Dirichlet problem

$$
\left\{\begin{array}{l}
-\Delta u=\lambda|u|^{q-2} u+|u|^{\gamma-2} u, \quad x \in \Omega, \\
\left.u\right|_{\partial \Omega}=0 .
\end{array}\right.
$$

Here $\Omega \subset \mathbb{R}^{N}$ is a bounded connected domain with a smooth boundary $\partial \Omega$. It is also assumed

$$
1<q<2<\gamma<2^{*}, \text { where } 2^{*}= \begin{cases}\frac{2 N}{N-2} & \text { as } N>2, \\ +\infty & \text { as } N \leqslant 2 .\end{cases}
$$

The main aim of the present work is to study the existence of nodal solutions to problem $(\mathcal{D})$. Equations of such kind appear in various fields of physics, for instance, in statistical mechanics, field theory, nonlinear optics and others (see [1]). Solutions to problem (D) can be also considered (cf. [2]) as stationary solutions of the associated boundary value problem for the nonlinear parabolic equation

$$
\begin{cases}u_{t}-\Delta u=\lambda|u|^{q-2} u+|u|^{\gamma-2} u, & (x, t) \in \Omega \times(0, T), \\ u=0, & (x, t) \in \partial \Omega \times(0, T), \\ u(0, x)=u_{0}(x), & x \in \Omega .\end{cases}
$$

Such problems appear in population dynamics (see [1]).

Many works were devoted to the existence of positive solutions of boundary value problem (D), see, for instance, [3], [4], [5], [6], [7]. For instance, in work [5], there was proven the

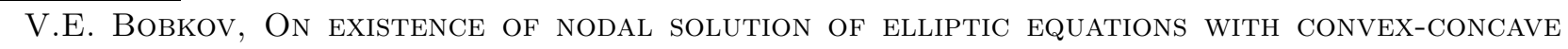
NONLINEARITIES.

(C) BobKov V.E. 2013.

The work is supported by RFBR (grant no. 13-01-00294-a).

Submitted March 5, 2012. 
existence of positive solutions $u_{0}$ which are the ground states of the corresponding Schrödinger equation [8], i.e.,

$$
I_{\lambda}\left(u_{0}\right) \leqslant I_{\lambda}(v),
$$

where $v \in W \backslash\{0\}$ is any solutions to problem $(\mathcal{D})$, and $I_{\lambda}$ is the associated energy functional, see below.

At the same time, employing Krasnoselski and Lyusternik-Schnirelmann topological methods, in the series of works ([3], [5], [9]), the existence of an infinitely many bound-state solutions $u_{k}$ of problem $(\mathcal{D})$ was shown, i.e., of the solutions satisfying

$$
I_{\lambda}\left(u_{0}\right)<I_{\lambda}\left(u_{k}\right) .
$$

However, this result gives no information on the structure of solutions, and moreover, since the employed methods are not constructive, it is difficult to use them for numeric calculations and analysis of such solutions. We note that finding of the bound-state solutions is also important for applications (see [10]).

Recently, there increased the interest to constructive finding bound-state solutions of nonlinear elliptic equations followed then by numerical analysis; it is reflected by appearance of a rather large number of publications on this subject (see, for instance, [11, [12, [13]). Basically, these results were obtained for coercive type equations for which direct variational methods are applicable. The situation for a more complicated nonlinearity, like concave-convex one, is little-studied. Concerning problem $(\mathcal{D})$, the main difficult is that the associated energy functional $I_{\lambda}(u)$ is not coercive and is not lower-semibounded. The geometry of the branches of such kind solutions is of complex structure. In particular, as it is known [5], equation (D) possesses multiple positive solutions and the bifurcations of turning points type.

In the present work we develop the fibering method $([14,15])$ and the spectral analysis by the fibering method ([16, 17]) for the set of nodal solutions.

Let us expound our result.

We consider weak solutions of problem (D), i.e., functions $u \in W \backslash\{0\}$ such that

$$
\int_{\Omega} \nabla u \nabla \phi d x=\lambda \int_{\Omega}|u|^{q-2} u \phi d x+\int_{\Omega}|u|^{\gamma-2} u \phi d x, \quad \forall \phi \in W \backslash\{0\},
$$

where $W=W_{0}^{1,2}(\Omega)$ is the standard Sobolev space being the closure of $C_{0}^{\infty}(\Omega)$ by the norm

$$
\|u\|=\left(\int_{\Omega}|\nabla u|^{2} d x\right)^{1 / 2} .
$$

It is easy to see that weak solutions to problem $(\mathcal{D})$ are critical points of the energy functional $I_{\lambda}$,

$$
I_{\lambda}(u)=\frac{1}{2} H(u)-\frac{\lambda}{q} G(u)-\frac{1}{\gamma} F(u)
$$

where

$$
H(u)=\int_{\Omega}|\nabla u|^{2} d x, \quad G(u)=\int_{\Omega}|u|^{q} d x, \quad F(u)=\int_{\Omega}|u|^{\gamma} d x .
$$

Together with $I_{\lambda}$, as in [5], we shall consider the functional $\mathcal{L}_{\lambda}$ on $W$ defined by the identity

$$
\mathcal{L}_{\lambda}(u)=H(u)-\lambda(q-1) G(u)-(\gamma-1) F(u),
$$

and we shall deal with the following characteristic value defined by the spectral parameter method [5],

$$
\lambda_{0}^{*}=\frac{q(\gamma-2)}{\gamma(2-q)}\left(\frac{\gamma(2-q)}{2(\gamma-q)}\right)^{\frac{\gamma-q}{\gamma-2}} \inf _{v \in W \backslash\{0\}}\left(\frac{H^{\frac{\gamma-q}{\gamma-2}}(v)}{G(v) F^{\frac{2-q}{\gamma-2}}(v)}\right) .
$$




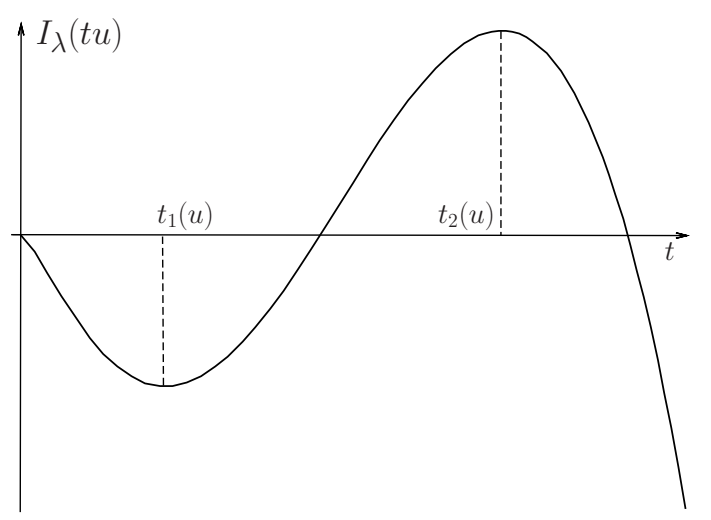

FiguRe 1.

We observe that $\mathcal{L}_{\lambda}(u)$ is determined (see [14]) via fibered functional $\tilde{I}_{\lambda}(t, u)=I_{\lambda}(t u)$ (the dependence of $I_{\lambda}(t u)$ on $t$ as $\lambda>0$ is given on fig. 1) by the formula

$$
\mathcal{L}_{\lambda}(u)=\left.\frac{\partial^{2}}{\partial t^{2}} I_{\lambda}(t u)\right|_{t=1}
$$

In what follows, while considering the functions $I_{\lambda}(t u)$ and $\mathcal{L}_{\lambda}(t u)$ w.r.t. $t$, we assume that $t>0$.

As it is known, each weak solution of problem (D) lies on the Nehari manifold, i.e., on the set

$$
\mathcal{N}_{\lambda}=\left\{u \in W \backslash\{0\}:\left.\frac{\partial}{\partial t} I_{\lambda}(t u)\right|_{t=1}=H(u)-\lambda G(u)-F(u)=0\right\} .
$$

By employing the fibering method, it is shown in [5] that if $\lambda<\lambda_{0}^{*}$, the Nehari manifold consists of two disjoint components. In the first component all weak solutions $u$ of problem $(\mathcal{D})$ satisfy inequality $\mathcal{L}_{\lambda}(u)<0$, while in the other $\mathcal{L}_{\lambda}(u)>0$.

Let $u \in W$. We introduce the functions $u_{+}=\max \{u, 0\} \geqslant 0$, and $u_{-}=\min \{u, 0\} \leqslant 0$. Then $u=u_{+}+u_{-}$and it can be shown that $u_{+} \in W$ and $u_{-} \in W$ (see Theorem 2, the proof is in [18]). We shall call the solutions $u$ obeying $u_{+} \neq 0$ and $u_{-} \neq 0$, as nodal solutions ([19]). Respectively, if $u \neq 0$, but $u_{+} \neq 0$ and $u_{-}=0$, or $u_{+}=0$ and $u_{-} \neq 0$, we shall call $u$ as sign-definite solution. Hereinafter, for an arbitrary $w \in W$, we shall assume that $w \neq 0$ if $\mu(\{x \in \Omega: w(x) \neq 0\}) \neq 0$, where $\mu$ is the Lebesgue measure on $\Omega$.

Our main result is the following theorem.

Theorem 1. Suppose $1<q<2<\gamma<2^{*}$ and value $\lambda_{0}^{*}$ is defined by variational problem (44). Then for each $\lambda \in\left(-\infty, \lambda_{0}^{*}\right)$ there exists a nodal solution $u_{\lambda}=u_{\lambda}^{+}+u_{\lambda}^{-}$of problem (D) such that

$$
u_{\lambda} \in \mathcal{N}_{\lambda}^{1}=\left\{u \in \mathcal{N}_{\lambda}: u_{+} \in \mathcal{N}_{\lambda}, \mathcal{L}_{\lambda}(u)<0, \mathcal{L}_{\lambda}\left(u_{+}\right)<0, \mathcal{L}_{\lambda}\left(u_{-}\right)<0\right\}
$$

At that, $u_{\lambda}$ is the ground state on the set $\mathcal{N}_{\lambda}^{1}$, i.e., $I_{\lambda}\left(u_{\lambda}\right) \leqslant I_{\lambda}(v)$ for each solution $v \in \mathcal{N}_{\lambda}^{1}$.

The paper is organized as follows. In Section 2 we provide auxiliary lemmas describing the properties of the energy functional $I_{\lambda}$ and its critical points as the parameter $\lambda$ ranges. In Section 3 we prove main result, Theorem 1, The appendix contains necessary technical statements. 


\section{ANALYSIS BY FIBERING METHOD}

We observe that the variational problem introduced above by identity (4) can be obtained from the following system of equations

$$
\left\{\begin{array}{l}
\frac{1}{2} t^{2} H(u)-\lambda \frac{1}{q} t^{q} G(u)-\frac{1}{\gamma} t^{\gamma} F(u)=0 \\
t H(u)-\lambda t^{q-1} G(u)-t^{\gamma-1} F(u)=0
\end{array}\right.
$$

that corresponds to the case $I_{\lambda}(t u)=0$ and $\frac{\partial}{\partial t} I_{\lambda}(t u)=0$ for an arbitrary function $u \in W \backslash\{0\}$. Solving this system w.r.t. $\lambda=\lambda(u)$ and $t=t(u)$, we obtain

$$
\begin{gathered}
\lambda(u)=\frac{q(\gamma-2)}{\gamma(2-q)}\left(\frac{\gamma(2-q)}{2(\gamma-q)}\right)^{\frac{\gamma-q}{\gamma-2}} \frac{H^{\frac{\gamma-q}{\gamma-2}}(u)}{G(v) F^{\frac{2-q}{\gamma-2}}(u)}, \\
t(u)=\left(\frac{\gamma(2-q)}{2(\gamma-q)} \frac{H(u)}{F(u)}\right)^{1 /(\gamma-2)} .
\end{gathered}
$$

Thus, following [5], from (6) we get the characteristic value

$$
\lambda_{0}^{*}=\inf _{u \in W \backslash\{0\}} \lambda(u) .
$$

Note that $t(u)>0$ and

$$
\begin{aligned}
\mathcal{L}_{\lambda(u)}(t(u) u) & =\left.t(u)^{2} \frac{\partial^{2}}{\partial t^{2}} I_{\lambda}(t u)\right|_{t=t(u)} \\
& =-t(u)^{2} \frac{(2-q)(\gamma-q)}{2} H(u)<0,
\end{aligned}
$$

i.e., $t(u)$ is the point of maximum of $I_{\lambda(u)}(t u)$ w.r.t. $t$.

Proposition 1. Suppose $1<q<2<\gamma<2^{*}, u \in W \backslash\{0\}$. Then there exists $\widetilde{\lambda}(u)>0$ such that

1) if $\lambda>\widetilde{\lambda}(u)$, the function $I_{\lambda}(t u)$ has no extrema w.r.t. $t$;

2) for each $\lambda \in(0, \widetilde{\lambda}(u))$, the function $I_{\lambda}(t u)$ has exactly one point of minimum $t_{1}(u)$ and one point of maximum $t_{2}(u)$ w.r.t. $t$, and moreover, $t_{1}(u)<t_{2}(u)$;

3) as $\lambda \leqslant 0$, the function $I_{\lambda}(t u)$ has exactly one point of maximum $t_{3}(u)$ w.r.t. $t$.

Proof. Let $u \in W \backslash\{0\}$. Then the equation $\frac{\partial}{\partial t} I_{\lambda}(t u)=0$ has at most two roots as $t>0$. Indeed, since $1<q<2<\gamma<2^{*}$, it follows from

$$
\frac{\partial}{\partial t} I_{\lambda}(t u)=t^{q-1}\left(t^{2-q} H(u)-\lambda G(u)-t^{\gamma-q} F(u)\right)=0
$$

that if $t>0$, the roots of the equation $\frac{\partial}{\partial t} I_{\lambda}(t u)=0$ coincide with that of

$$
\alpha_{\lambda}(t)=t^{\gamma-q} F(u)-t^{2-q} H(u)+\lambda G(u)=0 .
$$

Let us find the extrema of the function $\alpha_{\lambda}(t)$,

$$
\alpha_{\lambda}^{\prime}(t)=t^{1-q}\left((\gamma-q) t^{\gamma-2} F(u)-(2-q) H(u)\right)=0 .
$$

Since $t>0$, it implies

$$
(\gamma-q) t^{\gamma-2} F(u)-(2-q) H(u)=0 .
$$

The unique root of this equation is

$$
t=t(u)=\left(\frac{(2-q) H(u)}{(\gamma-q) F(u)}\right)^{1 / \gamma-2} .
$$


We note that if $t \in(0, t(u))$, then $\alpha_{\lambda}^{\prime}(t)<0$, and if $t>t(u)$, then $\alpha_{\lambda}^{\prime}(t)>0$, i.e., $t(u)$ is the point of minimum for the function $\alpha_{\lambda}(t)$ being its only extremum for $t>0$. In view of the form $\alpha_{\lambda}(t)$, it is obvious that for arbitrary $\lambda_{1}, \lambda_{2} \in \mathbb{R}$ such that $\lambda_{1}>\lambda_{2}$, the inequality $\alpha_{\lambda_{1}}(t)>\alpha_{\lambda_{2}}(t)$ holds true for each $t>0$. Let us find the value $\tilde{\lambda}=\tilde{\lambda}(u)$ for which the minimum of the function $\alpha_{\widetilde{\lambda}}(t)$ touches the axis $t$,

$$
\alpha_{\tilde{\lambda}}(t(u))=\left(\frac{(2-q) H(u)}{(\gamma-q) F(u)}\right)^{\frac{\gamma-q}{\gamma-2}} F(u)-\left(\frac{(2-q) H(u)}{(\gamma-q) F(u)}\right)^{\frac{2-q}{\gamma-2}} H(u)+\widetilde{\lambda} G=0 .
$$

It yields

$$
\widetilde{\lambda}=\frac{\gamma-2}{2-q}\left(\frac{2-q}{\gamma-q}\right)^{\frac{\gamma-q}{\gamma-2}} \frac{H^{\frac{\gamma-q}{\gamma-2}}(u)}{G(u) F^{\frac{2-q}{\gamma-2}}(u)} .
$$

Hence, if $\lambda>\widetilde{\lambda}$, then $\min _{t>0} \alpha_{\lambda}(t)>0$, i.e., the equation $\alpha_{\lambda}(t)=0$ has no roots. Therefore, as $\lambda>\widetilde{\lambda}$, the function $I_{\lambda}(t u)$ has no extrema w.r.t. $t$.

Suppose $\lambda \in(0, \widetilde{\lambda})$ (cf. fig. 2). Then $\alpha_{\lambda}(t)>0$ as $t \rightarrow 0$, and $\min _{t>0} \alpha_{\lambda}(t)<0$. Since $\alpha_{\lambda}^{\prime}(t)<0$ as $t \in(0, t(u))$, there exists the unique $t_{1}(u)>0$ such that $\alpha_{\lambda}\left(t_{1}(u)\right)=0$. At the same time, since $\alpha_{\lambda}^{\prime}(t)>0$ as $t>t(u)$ and $\alpha_{\lambda}(t) \rightarrow+\infty$ as $t \rightarrow+\infty$, there exists the unique $t_{2}(u)>0$ such that $\alpha_{\lambda}\left(t_{2}(u)\right)=0$. Thus, $t_{1}(u)$ and $t_{2}(u)$ are roots of the equation $\alpha_{\lambda}(t)=0$ and $t_{1}(u)<t_{2}(u)$. Moreover, since $-\alpha_{\lambda}^{\prime}(t)$ corresponds to $\frac{\partial^{2}}{\partial t^{2}} I_{\lambda}(t u), t_{1}(u)$ is the point of minimum and $t_{2}(u)$ is that of maximum for the function $I_{\lambda}(t u)$ w.r.t. $t$ (cf. fig. 1).

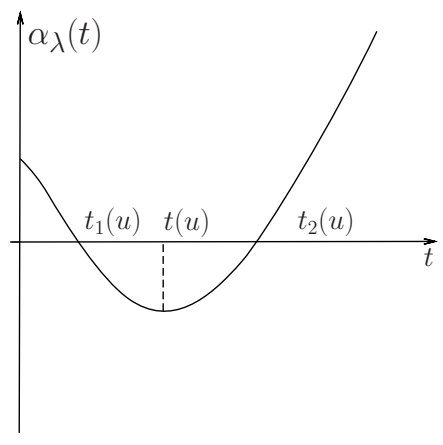

FiguRE 2.

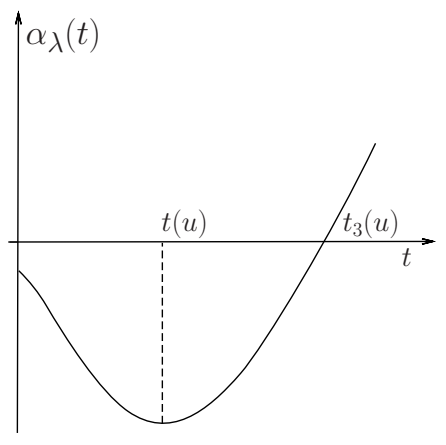

FiguRE 3.

Suppose $\lambda \leqslant 0$ (cf. fig. 3). Then $\alpha_{\lambda}(t) \leqslant 0$ as $t \rightarrow 0$ and $\min _{t>0} \alpha_{\lambda}(t)<0$. By a monotone decay of $\alpha_{\lambda}(t)$ as $t \in(0, t(u))$, on this segment $\alpha_{\lambda}(t)$ has no roots. In the same way, by a monotone growth of $\alpha_{\lambda}(t)$ as $t>t(u)$ and due to $\alpha_{\lambda}(t) \rightarrow+\infty$ as $t \rightarrow+\infty$, there exists the unique $t_{3}(u)>0$ such that $\alpha_{\lambda}\left(t_{3}(u)\right)=0$, i.e., $t_{3}(u)$ is the desired root and $t_{3}(u)$ is the point of maximum for the function $I_{\lambda}(t u)$ w.r.t. $t$.

Remark 1. It is easy to make sure that $\widetilde{\lambda}=\widetilde{\lambda}(u)$ determined by (17) solves the system

$$
\left\{\begin{array}{l}
t H(u)-\tilde{\lambda} t^{q-1} G(u)-t^{\gamma-1} F(u)=0 \\
2 H(u)-\tilde{\lambda} q t^{q-2} G(u)-\gamma t^{\gamma-2} F(u)=0
\end{array}\right.
$$

that appears in the case $\frac{\partial}{\partial t} I_{\tilde{\lambda}}(t u)=0, \frac{\partial^{2}}{\partial t^{2}} I_{\widetilde{\lambda}}(t u)=0$.

We introduce the following characteristic value

$$
\Lambda^{*}=\inf _{u \in W \backslash\{0\}} \widetilde{\lambda}(u),
$$

where $\tilde{\lambda}(u)$ is determined by (7). 
Proposition 2. Suppose $\lambda_{0}^{*}$ and $\Lambda^{*}$ are determined by variational problems (4) and (9), respectively. Then $\lambda_{0}^{*}<\Lambda^{*}$.

Proof. Suppose the opposite, $\lambda_{0}^{*} \geqslant \Lambda^{*}$. Comparing $\lambda_{0}^{*}$ and $\Lambda^{*}$, we rewrite the latter inequality as

$$
\frac{q}{\gamma}\left(\frac{\gamma}{2}\right)^{\frac{\gamma-q}{\gamma-2}} \geqslant 1
$$

Let $2=\alpha q, \gamma=2 \beta$, where by hypothesis of the lemma $\alpha, \beta>1$. Then inequality (10) can be written as

$$
\frac{1}{\alpha} \beta^{\frac{\alpha-1}{\alpha(\beta-1)}} \geqslant 1
$$

Taking logarithm of both sides, we get

$$
\frac{(\alpha-1) \ln \beta}{\alpha(\beta-1) \ln \alpha} \geqslant 1
$$

We estimate the logarithms by the inequality $\frac{t-1}{t} \leqslant \ln t \leqslant t-1$. We note that since $\alpha, \beta>1$, the strict inequalities $\frac{\alpha-1}{\alpha}<\ln \alpha<\alpha-1$ and $\frac{\beta-1}{\beta}<\ln \beta<\beta-1$ hold true. Hence,

$$
1 \leqslant \frac{(\alpha-1) \ln \beta}{\alpha(\beta-1) \ln \alpha}<1
$$

i.e., we obtain the contradiction. Thus, $\lambda_{0}^{*}<\Lambda^{*}$.

Let us prove the following statement.

Lemma 1. Suppose $1<q<2<\gamma<2^{*}, \lambda<\Lambda^{*}$, and $u \in \mathcal{N}_{\lambda}$. Then

1. $\mathcal{L}_{\lambda}(u) \neq 0$,

2. $I_{\lambda}(u) \rightarrow+\infty$ as $\|u\| \rightarrow+\infty$, i.e., the functional $I_{\lambda}$ is coercive on $\mathcal{N}_{\lambda}$.

Proof. Let $1<q<2<\gamma<2^{*}, \lambda<\Lambda^{*}$, and $u \in \mathcal{N}_{\lambda}$.

1) Suppose the opposite, $\mathcal{L}_{\lambda}(u)=0$. Then the function $u$ satisfies system (8) for $t=1$ and $\lambda=\widetilde{\lambda}(u)$ determined by (17). But in this case $\Lambda^{*} \leqslant \lambda=\widetilde{\lambda}(u)$ that contradicts the hypothesis. Thus, $\mathcal{L}_{\lambda}(u) \neq 0$.

2) Let us prove the coercivity of the functional $I_{\lambda}$ on $\mathcal{N}_{\lambda}$. Suppose $u \in \mathcal{N}_{\lambda}$, i.e., the condition $\left.\frac{\partial}{\partial t} I_{\lambda}(t u)\right|_{t=1}=0$ holds true. Then the functional $I_{\lambda}$ on $\mathcal{N}_{\lambda}$ can be written as

$$
I_{\lambda}(u)=\frac{\gamma-2}{2 \gamma} H(u)-\lambda \frac{\gamma-q}{\gamma q} G(u) .
$$

If $\lambda>0$, by the embedding theorem it implies the estimate

$$
I_{\lambda}(u)>\frac{\gamma-2}{2 \gamma} H(u)-\lambda \frac{\gamma-q}{\gamma q} C_{q} H(u)^{q / 2},
$$

where $C_{q}=C_{q}(q, \gamma, \Omega)>0$.

If $\lambda \leqslant 0$, we estimate (111) as follows,

$$
I_{\lambda}(u) \geqslant \frac{\gamma-2}{2 \gamma} H(u)
$$

Then in both cases $I_{\lambda}(u) \rightarrow+\infty$ as $H(u)=\|u\|^{2} \rightarrow+\infty$. That is, the functional $I_{\lambda}$ is coercive on $\mathcal{N}_{\lambda}$.

Corollary 1. Suppose $1<q<2<\gamma<2^{*}$ and $\lambda<\Lambda^{*}$. If $u \in W \backslash\{0\}$ is such that $u_{+} \in \mathcal{N}_{\lambda}$ $\left(u_{-} \in \mathcal{N}_{\lambda}\right)$, then $\mathcal{L}_{\lambda}\left(u_{+}\right) \neq 0\left(\mathcal{L}_{\lambda}\left(u_{-}\right) \neq 0\right)$. 
Proof. Let $u \in W \backslash\{0\}$ and $u_{+} \in \mathcal{N}_{\lambda}$. Denote $\Omega_{+}:=\operatorname{supp} u_{+}$, then it is obvious that

$$
u_{+}(x)= \begin{cases}u(x) & , \quad x \in \Omega_{+} \\ 0 & x \in \Omega \backslash \Omega_{+} .\end{cases}
$$

Hence, for each test function $u \in W \backslash\{0\}$ of the minimization problem (9), the function $u_{+} \in$ $W$ is a test one as well once $u_{+} \neq 0$. As it is known, additional restrictions posed for the minimization problem do not lessen the infimum and thus

$$
\Lambda^{*}=\Lambda_{\Omega}^{*}=\inf _{u \in W \backslash\{0\}} \tilde{\lambda}(u) \leqslant \inf _{\substack{u \in W \backslash\{0\}: \\ u(x)=0, x \in \Omega \backslash \Omega_{+}}} \tilde{\lambda}(u)=\Lambda_{\Omega_{+}}^{*} .
$$

By the assumption, $\lambda<\Lambda^{*}$. Hence, $\lambda<\Lambda^{*} \leqslant \Lambda_{\Omega_{+}}^{*}$ and by Lemma 1, $\mathcal{L}_{\lambda}\left(u_{+}\right) \neq 0$.

Remark 2. Due to Proposition 2, the results of Lemma 1 and its Corollary remain true for $\lambda<\lambda_{0}^{*}$.

In what follows we shall make use of certain properties of $I_{\lambda}(u)$ on the Nehari set.

Lemma 2. Let $1<q<2<\gamma<2^{*}$ and $\lambda<\lambda_{0}^{*}$. If $u \in \mathcal{N}_{\lambda}$ and $\mathcal{L}_{\lambda}(u)<0$, then

1) $I_{\lambda}(u)>0$,

2) $t=1$ is the point of the global maximum of the function $I_{\lambda}(t u)$ w.r.t. $t$ as $t>0$,

3) $\|u\|>\delta>0$, where $\delta$ is independent of $u$.

Proof. Let $u \in \mathcal{N}_{\lambda}, \mathcal{L}_{\lambda}(u)<0$ and $\lambda<\lambda_{0}^{*}$.

1) We note that $\lambda<\lambda_{0}^{*} \leqslant \lambda(u)$, where $\lambda(u)$ is determined by identity (6). Then

$$
I_{\lambda}(u)=\frac{1}{2} H(u)-\frac{\lambda}{q} G(u)-\frac{1}{\gamma} F(u)>\frac{1}{2} H(u)-\frac{\lambda(u)}{q} G(u)-\frac{1}{\gamma} F(u)=0 .
$$

2) By Proposition 1, $t=1$ is the unique point of local maximum of the function $I_{\lambda}(t u)$ w.r.t. $t$ as $t>0$, and $I_{\lambda}(u)>0$. At that, on the boundary of the domain $(0,+\infty)$ we have

$$
\begin{aligned}
& I_{\lambda}(t u) \rightarrow 0 \quad \text { as } \quad t \rightarrow 0, \\
& I_{\lambda}(t u) \rightarrow-\infty \quad \text { as } \quad t \rightarrow+\infty .
\end{aligned}
$$

Therefore, $t=1$ is the point of the global maximum of $I_{\lambda}(t u)$ w.r.t. $t$.

3) We write the conditions $\left.\frac{\partial}{\partial t} I_{\lambda}(t u)\right|_{t=1}=0$ and $\left.\frac{\partial^{2}}{\partial t^{2}} I_{\lambda}(t u)\right|_{t=1}<0$ as the system

$$
\left\{\begin{array}{l}
H(u)-\lambda G(u)-F(u)=0, \\
H(u)-\lambda(q-1) G(u)-(\gamma-1) F(u)<0 .
\end{array}\right.
$$

We express $\lambda G(v)$ by the first equation and substitute it into the inequality. Then, by Sobolev embedding theorem, we get the chain of inequalities,

$$
\frac{2-q}{\gamma-q} H(u)<F(u)<C_{\gamma} H(u)^{\gamma / 2}, \quad C_{\gamma}=C_{\gamma}(q, \gamma, \Omega)>0
$$

It implies

$$
H(u)>\left(\frac{2-q}{(\gamma-q) C_{\gamma}}\right)^{\frac{2}{\gamma-2}}=\delta^{2}(q, \gamma, \Omega)=\delta^{2}>0 .
$$

Thus, $\|u\|=H(u)^{1 / 2}>\delta>0$.

Remark 3. It is obvious that the results of Lemma回 remain true for $u, u_{+}, u_{-}$if $u \in \mathcal{N}_{\lambda}^{1}$. 
We note that it follows from Theorem 2 (see Appendix) that if $u \in W$, then $u_{+} \in W$ and $u_{-} \in W$. Moreover, for the representation $u=u_{+}+u_{-}$, the identity

$$
I_{\lambda}(u)=I_{\lambda}\left(u_{+}\right)+I_{\lambda}\left(u_{-}\right)
$$

holds true that also follows from Theorem 2. This identity implies easily the following ones,

$$
\begin{gathered}
\frac{\partial}{\partial t} I_{\lambda}(t u)=\frac{\partial}{\partial t} I_{\lambda}\left(t u_{+}\right)+\frac{\partial}{\partial t} I_{\lambda}\left(t u_{-}\right), \\
\mathcal{L}_{\lambda}(u)=\mathcal{L}_{\lambda}\left(u_{+}\right)+\mathcal{L}_{\lambda}\left(u_{-}\right) .
\end{gathered}
$$

Let us show now that as $\lambda<\lambda_{0}^{*}$, the set $\mathcal{N}_{\lambda}^{1}$ is non-empty. We take an arbitrary subdomain $\Omega_{1} \subset \Omega$ and a function $u_{1} \in W \backslash\{0\}$ such that $\operatorname{supp} u_{1}=\bar{\Omega}_{1}$. By Proposition 1, there exists $t_{1}\left(u_{1}\right)>0$ such that $\left.\frac{\partial}{\partial t} I_{\lambda}\left(t u_{1}\right)\right|_{t=t_{1}\left(u_{1}\right)}=0$ and $\mathcal{L}_{\lambda}\left(t_{1}\left(u_{1}\right) u_{1}\right)<0$. We choose now some subdomain $\Omega_{2} \subset \Omega$ such that $\Omega_{1} \cap \Omega_{2}=\emptyset$ and a function $u_{2} \in W \backslash\{0\}$ such that $\operatorname{supp} u_{2}=\bar{\Omega}_{2}$. Then there exists $t_{2}\left(u_{2}\right)>0$ such that $\left.\frac{\partial}{\partial t} I_{\lambda}\left(t u_{2}\right)\right|_{t=t_{2}\left(u_{2}\right)}=0$ and $\mathcal{L}_{\lambda}\left(t_{2}\left(u_{2}\right) u_{2}\right)<0$. We denote $v_{+}=t_{1}\left(u_{1}\right) u_{1}, \quad v_{-}=-t_{2}\left(u_{2}\right) u_{2}$ and $v=v_{+}+v_{-}$. Then

$$
\begin{gathered}
\left.\frac{\partial}{\partial t} I_{\lambda}(t v)\right|_{t=1}=\left.\frac{\partial}{\partial t} I_{\lambda}\left(t v_{+}\right)\right|_{t=1}+\left.\frac{\partial}{\partial t} I_{\lambda}\left(t v_{-}\right)\right|_{t=1}=0 \\
\mathcal{L}_{\lambda}(v)=\mathcal{L}_{\lambda}\left(v_{+}\right)+\mathcal{L}_{\lambda}\left(v_{-}\right)<0 .
\end{gathered}
$$

Hence, we have found a function $v \in W \backslash\{0\}$ such that $v \in \mathcal{N}_{\lambda}^{1}$, i.e., $\mathcal{N}_{\lambda}^{1} \neq \emptyset$.

Consider the following minimization problem with restrictions,

$$
\left\{\begin{array}{l}
I_{\lambda}(u) \rightarrow \min , \\
u \in \mathcal{N}_{\lambda}^{1} .
\end{array}\right.
$$

Lemma 3. Suppose $1<q<2<\gamma<2^{*}$ and $\lambda<\lambda_{0}^{*}$. If $u \in \mathcal{N}_{\lambda}^{1}$ is a solution to minimization problem (14), then $u$ is a critical point of $I_{\lambda}$ on $W \backslash\{0\}$, i.e.,

$$
\left\langle D_{u} I_{\lambda}(u), \phi\right\rangle=0, \quad \forall \phi \in W .
$$

Proof. Let $u \in \mathcal{N}_{\lambda}^{1}$ be a solution to minimization problem (14), i.e., $\beta=I_{\lambda}(u)=\inf \left\{I_{\lambda}(v): v \in \mathcal{N}_{\lambda}^{1}\right\}$. Suppose the opposite, $D_{u} I_{\lambda}(u) \neq 0$.

Since $\lambda<\lambda_{0}^{*}$, by Lemma 2, $t=1$ is the point of the global maximum for the function $I_{\lambda}(t u)$ w.r.t. $t$. Moreover, Remark 3 implies that $t=1$ is also the point of the global minimum of the functions $I_{\lambda}\left(t u_{+}\right)$and $I_{\lambda}\left(t u_{-}\right)$w.r.t. $t$. Therefore,

$$
\begin{aligned}
I_{\lambda}\left(s u_{+}+t u_{-}\right)=I_{\lambda}\left(s u_{+}\right)+I_{\lambda}\left(t u_{-}\right) & < \\
I_{\lambda}\left(u_{+}\right)+I_{\lambda}\left(u_{-}\right) & =I_{\lambda}\left(u_{+}+u_{-}\right)=I_{\lambda}(u)
\end{aligned}
$$

for each $(s, t) \in \mathbb{R}_{+}^{2} \backslash\{1,1\}$.

Since by the assumption $D_{u} I_{\lambda}(u) \neq 0$, due to the continuity of the functional $D_{u} I_{\lambda}$, there exist $\alpha, \delta>0$ such that $\left\|D_{u} I_{\lambda}(v)\right\| \geqslant \alpha$ as $v \in U_{3 \delta}(u)=\{w \in W:\|u-w\|<3 \delta\}$.

We introduce the function

$$
\begin{gathered}
g: A=\left(\frac{1-t_{1}\left(u_{+}\right)}{2}, \frac{1+t_{1}\left(u_{+}\right)}{2}\right) \times\left(\frac{1-t_{1}\left(u_{-}\right)}{2}, \frac{1+t_{1}\left(u_{-}\right)}{2}\right) \rightarrow W, \\
g(s, t)=s u_{+}+t u_{-} .
\end{gathered}
$$

We recall that $t_{1}\left(u_{+}\right)$and $t_{1}\left(u_{-}\right)$are respectively the points of minimum and maximum of the functions $I_{\lambda}\left(t u_{+}\right)$and $I_{\lambda}\left(t u_{-}\right)$w.r.t. $t$.

Proposition 1 and the condition $\lambda<\lambda_{0}^{*}$ follow that $t_{1}\left(u_{+}\right), t_{1}\left(u_{-}\right)<1$ and hence $A \neq \emptyset$. Moreover, it follows from (15) that

$$
\beta_{0}:=\max _{(s, t) \in \partial A} I_{\lambda}(g(s, t))<\beta .
$$


Denote $\varepsilon:=\min \left\{\frac{\beta-\beta_{0}}{2}, \frac{\alpha \delta}{8}\right\}$ and $S=U_{\delta}(u)$. Then the deformation lemma (see Theorem 3 in Appendix) implies the existence of a homotopy $\eta$ such that $\eta_{1}:=\eta(1, \cdot): W \rightarrow W$ satisfies

1) $\eta_{1}(v)=v$ if $I_{\lambda}(v) \leqslant \beta-2 \varepsilon$,

2) $\eta_{1}\left(\left\{v \in S: I_{\lambda}(v) \leqslant \beta+\varepsilon\right\}\right) \subset\left\{v \in W: I_{\lambda}(v) \leqslant \beta-\varepsilon\right\}$,

3) $I_{\lambda}\left(\eta_{1}(v)\right) \leqslant I_{\lambda}(v)$ for each $v \in W$.

It follows from 2 ) that

$$
\max _{\{(s, t) \in A: g(s, t) \in S\}} I_{\lambda}\left(\eta_{1}(g(s, t))\right)<\beta .
$$

On the other hand, 3) and (15) imply

$$
\max _{\{(s, t) \in A: g(s, t) \notin S\}} I_{\lambda}\left(\eta_{1}(g(s, t))\right) \leqslant \max _{\{(s, t) \in A: g(s, t) \notin S\}} I_{\lambda}(g(s, t))<\beta .
$$

For the sake of convenience we denote

$$
f:=\eta_{1}(g(s, t))
$$

Then it follows from 1$)$ that $f(s, t)=g(s, t)$ as $(s, t) \in \partial A$ by the choice of $\varepsilon$. Consider the mapping

$$
\psi: A \rightarrow \mathbb{R}^{2}, \quad \psi(s, t):=\left(Q_{\lambda}\left(f(s, t)_{+}\right), Q_{\lambda}\left(f(s, t)_{-}\right)\right),
$$

where $Q_{\lambda}(s u):=\left\langle D_{u} I_{\lambda}(s u), u\right\rangle=\left.s \frac{\partial}{\partial t} I_{\lambda}(t u)\right|_{t=s}$. Note that $\psi(s, t)=(0,0)$ if and only if $f(s, t)_{+}, f(s, t)_{-} \in \mathcal{N}_{\lambda}$.

Since $f=g$ as $(s, t) \in \partial A$, then

$$
\psi(s, t)=\left(Q_{\lambda}\left(s u_{+}\right), Q_{\lambda}\left(t u_{-}\right)\right), \quad(s, t) \in \partial A .
$$

At that,

$$
\begin{aligned}
& Q_{\lambda}\left(\frac{1-t_{1}\left(u_{+}\right)}{2} u_{+}\right)>0>Q_{\lambda}\left(\frac{1+t_{1}\left(u_{+}\right)}{2} u_{+}\right), \\
& Q_{\lambda}\left(\frac{1-t_{1}\left(u_{-}\right)}{2} u_{-}\right)>0>Q_{\lambda}\left(\frac{1+t_{1}\left(u_{-}\right)}{2} u_{-}\right) .
\end{aligned}
$$

Then Theorem 4 in Appendix yields the existence a point $\left(s_{0}, t_{0}\right) \in A$ such that $\psi\left(s_{0}, t_{0}\right)=$ $(0,0)$, and therefore, $f\left(s_{0}, t_{0}\right)_{+}, f\left(s_{0}, t_{0}\right)_{-} \in \mathcal{N}_{\lambda}$. Moreover, it follows from (18), (19) and Proposition 1 that $\mathcal{L}_{\lambda}\left(f\left(s_{0}, t_{0}\right)_{+}\right)<0$ and $\mathcal{L}_{\lambda}\left(f\left(s_{0}, t_{0}\right)_{-}\right)<0$, since there exists the unique point of maximum of the functions $I_{\lambda}\left(z f\left(s_{0}, t_{0}\right)_{+}\right)$and $I_{\lambda}\left(z f\left(s_{0}, t_{0}\right)_{-}\right)$w.r.t. $z$ as $z>0$.

Hence, $f\left(s_{0}, t_{0}\right) \in \mathcal{N}_{\lambda}^{1}$, i.e., $f\left(s_{0}, t_{0}\right)$ is an admissible function for minimization problem (14). Moreover, it follows from (16) and (17) that

$$
I_{\lambda}\left(f\left(s_{0}, t_{0}\right)\right)<\beta=\inf \left\{I_{\lambda}(v): v \in \mathcal{N}_{\lambda}^{1}\right\},
$$

i.e., we get the contradiction. Thus, $D_{u} I_{\lambda}(u)=0$, i.e., $u$ is a critical point of $I_{\lambda}$ on $W \backslash\{0\}$.

\section{EXISTENCE OF NODAL SOLUTIONS}

We shall assume that $1<q<2<\gamma<2^{*}$ and $\lambda<\lambda_{0}^{*}$. Let

$$
c_{1}=\inf \left\{I_{\lambda}(v): v \in \mathcal{N}_{\lambda}^{1}\right\}
$$

and $u_{n} \in \mathcal{N}_{\lambda}^{1}$ is a minimizing sequence, i.e., $I_{\lambda}\left(u_{n}\right) \rightarrow c_{1}$. At that, by Lemma 2 , $c_{1} \geqslant 0$. Then, by coercivity of $I_{\lambda}$ on $\mathcal{N}_{\lambda}$ (see Lemma 1), the sequence $u_{n}$ is bounded in $W$. By the reflexivity of the space $W$ and the Eberlein-Šmulain theorem [20], there exists $u, v, w \in W$ such that

$$
u_{n} \rightarrow u, \quad\left(u_{n}\right)_{+} \rightarrow v, \quad\left(u_{n}\right)_{-} \rightarrow w \text { weakly in } W .
$$

Moreover, keeping the same indexing by $n$, by Sobolev embedding theorem we get

$$
\begin{aligned}
& u_{n} \rightarrow u, \quad\left(u_{n}\right)_{+} \rightarrow v, \quad\left(u_{n}\right)_{-} \rightarrow w \text { in } L^{\gamma}, \\
& u_{n} \rightarrow u, \quad\left(u_{n}\right)_{+} \rightarrow v, \quad\left(u_{n}\right)_{-} \rightarrow w \text { in } L^{q},
\end{aligned}
$$


since $q<\gamma<2^{*}$.

By Lemma 4 it is known that as $r=q$ and $r=\gamma$, the mapping $h: L^{r} \rightarrow L^{r}\left(u \rightarrow u_{+}\right)$is continuous and it thus follows from (21) that $u_{+}=v \geqslant 0$ and $u_{-}=w \leqslant 0$. Let us show that $u$ changes sign, i.e., $u_{+}>0$ and $u_{-}<0$. Since $u_{n} \in \mathcal{N}_{\lambda}^{1}$, Lemma 2 implies

$$
\lambda \int_{\Omega}(u)_{+}^{q}+\int_{\Omega}(u)_{+}^{\gamma} d x=\lim _{n \rightarrow \infty}\left(\lambda \int_{\Omega}\left(u_{n}\right)_{+}^{q} d x+\int_{\Omega}\left(u_{n}\right)_{+}^{\gamma} d x\right)=\lim _{n \rightarrow \infty}\left\|\left(u_{n}\right)_{+}\right\|^{2}>0 .
$$

Therefore, $u_{+}>0$. In the same way one can show that $u_{-}<0$.

Let us show now that $\left(u_{n}\right)_{+} \rightarrow u_{+}$in $W$. The weak convergence of $\left(u_{n}\right)_{+}$to $u_{+}$in $W$ and the weak lower semicontinuity of the norm of the space $W$ yield $\left\|u_{+}\right\|^{2} \leqslant \liminf _{n \rightarrow \infty}\left\|\left(u_{n}\right)_{+}\right\|^{2}$. Let us show that the identity holds true. Suppose $\left\|u_{+}\right\|^{2}<\liminf _{n \rightarrow \infty}\left\|\left(u_{n}\right)_{+}\right\|^{2}$. Then

$$
\left\|u_{+}\right\|^{2}-\lambda G\left(u_{+}\right)-F\left(u_{+}\right)<\liminf _{n \rightarrow \infty}\left(\left\|\left(u_{n}\right)_{+}\right\|^{2}-\lambda G\left(\left(u_{n}\right)_{+}\right)-F\left(\left(u_{n}\right)_{+}\right)\right)=0 .
$$

Let $\lambda \in\left(0, \lambda_{0}^{*}\right)$. By Proposition 1, there exist $\alpha=t_{2}\left(u_{+}\right)>0$ and $\beta=t_{2}\left(u_{-}\right)>0$ such that

$$
\begin{array}{cc}
\left.\frac{\partial}{\partial t} I_{\lambda}\left(t u_{+}\right)\right|_{t=\alpha}=0, & \left.\frac{\partial}{\partial t} I_{\lambda}\left(t u_{-}\right)\right|_{t=\beta}=0, \\
\left.\frac{\partial^{2}}{\partial t^{2}} I_{\lambda}\left(t u_{+}\right)\right|_{t=\alpha}<0, & \left.\frac{\partial^{2}}{\partial t^{2}} I_{\lambda}\left(t u_{-}\right)\right|_{t=\beta}<0 .
\end{array}
$$

In this case $\left.\frac{\partial}{\partial t} I_{\lambda}\left(t\left(\alpha u_{+}+\beta u_{-}\right)\right)\right|_{t=1}=0$. Then, by the assumption,

$$
\begin{aligned}
I_{\lambda}\left(\alpha u_{+}+\beta u_{-}\right) & <\liminf _{n \rightarrow \infty}\left(I_{\lambda}\left(\alpha\left(u_{n}\right)_{+}+\beta\left(u_{n}\right)_{-}\right)\right) \\
& =\liminf _{n \rightarrow \infty}\left(I_{\lambda}\left(\alpha\left(u_{n}\right)_{+}\right)+I_{\lambda}\left(\beta\left(u_{n}\right)_{-}\right)\right) .
\end{aligned}
$$

In its turn, by Remark 3,

$$
\liminf _{n \rightarrow \infty}\left(I_{\lambda}\left(\alpha\left(u_{n}\right)_{+}\right)+I_{\lambda}\left(\beta\left(u_{n}\right)_{-}\right)\right) \leqslant \liminf _{n \rightarrow \infty}\left(I_{\lambda}\left(\left(u_{n}\right)_{+}\right)+I_{\lambda}\left(\left(u_{n}\right)_{-}\right)\right)
$$

since $u_{n} \in \mathcal{N}_{\lambda}^{1}$, i.e., $t=1$ is the point of the global maximum of the functions $I_{\lambda}\left(t u_{+}\right)$and $I_{\lambda}\left(t u_{-}\right)$w.r.t. $t$. At the same time,

$$
\liminf _{n \rightarrow \infty}\left(I_{\lambda}\left(\left(u_{n}\right)_{+}\right)+I_{\lambda}\left(\left(u_{n}\right)_{-}\right)\right)=\liminf _{n \rightarrow \infty} I_{\lambda}\left(u_{n}\right)=\inf _{\mathcal{N}_{\lambda}^{1}} I_{\lambda}=c_{1} .
$$

Thus, (23), (24), (25) imply $I_{\lambda}\left(\alpha u_{+}+\beta u_{-}\right)<c_{1}$ that contradict the assumption. We obtain the contradiction and therefore $\left(u_{n}\right)_{+} \rightarrow u_{+},\left(u_{n}\right)_{-} \rightarrow u_{-}$in $W$ and $\alpha=\beta=1$. Similar result holds as $\lambda \leqslant 0$.

Hence, $u \in \mathcal{N}_{\lambda}^{1}$ and

$$
I_{\lambda}(u)=\inf \left\{I_{\lambda}(v): v \in \mathcal{N}_{\lambda}^{1}\right\} .
$$

Thus, $u$ is a solution of ground state type on the set $\mathcal{N}_{\lambda}^{1}$.

\section{APPENDIX}

We provide necessary statements.

Theorem 2. Let $\Omega \in \mathbb{R}^{N}, u \in W_{0}^{1, p}(\Omega), 0 \leqslant p \leqslant \infty$. Then $\max \{u, 0\} \in W_{0}^{1, p}(\Omega)$ and for $1 \leqslant i \leqslant N$ the identity

$$
\frac{\partial}{\partial x_{i}} \max \{u, 0\}= \begin{cases}\frac{\partial u}{\partial x_{i}}, & \text { in }\{x \in \Omega: u(x)>0\} \\ 0, & \text { in }\{x \in \Omega: u(x) \leqslant 0\}\end{cases}
$$

holds true in the generalized sense.

Proof. See [18]. 
Corollary 2. Let $\Omega \in \mathbb{R}^{N}, u \in W_{0}^{1, p}(\Omega), 0 \leqslant p \leqslant \infty$. Then

$$
\frac{\partial u}{\partial x_{i}}=0 \text { a.e. in } E=\{x \in \Omega: u=0\}, \quad 1 \leqslant i \leqslant N .
$$

Define the mapping $h: L^{r} \rightarrow L^{r}, r \geqslant 1$, by the formula $h(u)=\max \{u, 0\}$.

Lemma 4. The mapping $h$ is continuous.

Proof. Let $u \in L^{r}(\Omega)$. Then it is obvious that $u_{+} \in L^{r}(\Omega)$ and $u_{-} \in L^{r}(\Omega)$. We note that almost everywhere in $\Omega$ the mapping $h$ can be represented as $h(u)=j(u) u$, where $j(u)=1$ if $u \geqslant 0$ and $j(u)=0$ if $u<0$. Suppose $u_{n} \rightarrow u$ in $L^{r}(\Omega)$. Then there exists a subsequence $u_{n_{k}}$ such that $u_{n_{k}} \rightarrow u$ alsmot everywhere in $\Omega$. For the sake of brevity and without loss of generality, we keep the previous indexing by $n$ not passing to the indexing of the subsequence. Then

$$
\begin{aligned}
\left\|h\left(u_{n}\right)-h(u)\right\|_{r}^{r} & =\int_{\Omega}\left|h\left(u_{n}\right)-h(u)\right|^{r} d x= \\
& =\int_{\Omega}\left|j\left(u_{n}\right)\left(u_{n}-u\right)+\left(j\left(u_{n}\right)-j(u)\right) u\right|^{r} d x .
\end{aligned}
$$

We observe that since $\varphi(s)=s^{r}$ is a convex function as $r \geqslant 1$ and $s \geqslant 0$, by employing Jensen's inequality $\left(s_{1}+s_{2}\right)^{r} \leqslant 2^{r-1}\left(s_{1}^{r}+s_{2}^{r}\right)$ we obtain

$$
\left\|h\left(u_{n}\right)-h(u)\right\|_{r}^{r} \leqslant 2^{r-1}\left(\int_{\Omega}\left|\left(u_{n}-u\right)\right|^{r} d x+\int_{\Omega}\left|\left(j\left(u_{n}\right)-j(u)\right) u\right|^{r} d x\right) .
$$

The first integral converges to 0 , since $u_{n} \rightarrow u$ in $L^{r}(\Omega)$. On the other hand, for a.e. $x \in \Omega$, either $j\left(u_{n}\right) \rightarrow j(u)=0$ or $j\left(u_{n}\right) \rightarrow j(u)=1$. It implies

$$
\int_{\Omega}\left|\left(j\left(u_{n}\right)-j(u)\right) u\right|^{r} d x \leqslant \sup _{x \in \Omega}\left(j\left(u_{n}\right)-j(u)\right) \int_{\Omega}|u|^{r} d x \rightarrow 0
$$

by $u \in L^{r}(\Omega)$. Thus, $\left\|h\left(u_{n}\right)-h(u)\right\|_{r} \rightarrow 0$. Therefore, $h \in C\left(L^{r} ; L^{r}\right)$.

Corollary 3. Employing similar arguments, one can get easily $h \in C(W ; W)$.

The next theorem is a version of the deformation lemma.

Theorem 3 (Deformation lemma). Let $X$ be a Banach space, $I \in \mathcal{C}^{1}(X, \mathbb{R}), S \subset X, c \in \mathbb{R}$, $\varepsilon>0, \delta>0$ be such that

$$
\left\|D_{u} I(u)\right\|_{X^{*}} \geqslant \frac{8 \varepsilon}{\delta}, \quad \forall u \in I^{-1}([c-2 \varepsilon, c+\varepsilon]) \cap S_{2 \delta}
$$

where $S_{2 \delta}=\{v \in X: \operatorname{dist}(v, S) \leqslant 2 \delta\}$. Then there exists a homotopy $\eta \in \mathcal{C}([0,1] \times X, X)$ such that

- if $t=0$ or $u \notin I^{-1}([c-2 \varepsilon, c+\varepsilon]) \cap S_{2 \delta}$, then $\eta(t, u)=u$;

- $\eta(1,\{v \in S: I(v) \leqslant c+\varepsilon\}) \subset\{v \in W: I \leqslant c-\varepsilon\}$;

- $\eta(1, \cdot)$ defines a homeomorphism $X \rightarrow X$ for each $t \in[0,1]$;

- $\|\eta(t, u)-u\|_{X} \leqslant \delta$ for each $u \in X, t \in[0,1]$;

- $I(\eta(\cdot, u))$ does not grow for each $u \in X$;

- $I(\eta(t, u))<c$ for each $u \in I^{-1}((-\infty, c]) \cap S_{\delta}, t \in[0,1]$.

Proof. See [21], Lemma 1.4.

The next theorem is a two-dimensional version of Miranda theorem (see, for instnace, [22]). 
Theorem 4 (Miranda, 1940). Suppose $A=\left\{x \in \mathbb{R}^{2}: a_{1} \leqslant x_{1} \leqslant b_{1}, a_{2} \leqslant x_{2} \leqslant b_{2}\right\}$, the mapping

$$
\psi=\left(\psi_{1}, \psi_{2}\right): A \rightarrow \mathbb{R}^{2}
$$

is continuous, and

$$
\begin{array}{ll}
\psi_{1}\left(a_{1}, x_{2}\right) \geqslant 0 \geqslant \psi_{1}\left(b_{1}, x_{2}\right), & \forall x_{2} \in\left(a_{2}, b_{2}\right), \\
\psi_{2}\left(x_{1}, a_{2}\right) \geqslant 0 \geqslant \psi_{2}\left(x_{1}, b_{2}\right), & \forall x_{1} \in\left(a_{1}, b_{1}\right) .
\end{array}
$$

Then there exists a point $\left(x_{1}^{0}, x_{2}^{0}\right) \in A$ such that $\psi\left(x_{1}^{0}, x_{2}^{0}\right)=(0,0)$.

Proof. See [22].

\section{BIBLIOGRAPHY}

1. H. Berestycki, P.L. Lions. Nonlinear scalar field equations, I. Existence of a ground state // Arch. Ration. Mech. An. 1983. V. 82, No. 4. P. 313-345.

2. T. Cazenave, F. Dickstein, M. Escobedo. A semilinear heat equation with concave-convex nonlinearity // Rend. Matem. Ser. VII. 1999. V. 19. P. 211-242.

3. A. Ambrosetti, H. Brezis, G. Cerami. Combined effects of concave and convex nonlinearities in some elliptic problems // J. Funct. Anal. 1994. V. 122, No. 2. P. 519-543.

4. T. Bartsch, M. Willem. On an elliptic equation with concave and convex nonlinearities // Proc. Amer. Math. Soc. 1995. V. 123, No. 11. P. 3555-3561.

5. Ya. Il'yasov. On nonlocal existence results for elliptic equations with convex-concave nonlinearities // Nonlinear Anal.-Theor. 2005. V. 61, No. 1-2. P. 211-236.

6. L. Boccardo, M. Escobedo, I. Peral. A Dirichlet problem involving critical exponents // Nonlinear Anal.-Theor. 1995. V. 24, No. 11. P. 1639-1648.

7. G. Azorero, J. Manfredi, I. Alonso. Sobolev versus Hlder local minimizes and global multiplicity for some quasilinear elliptic equations // Commun. Contemp. Math. 2000. V. 2, No. 3. P. 385-404.

8. S. Coleman, V. Glazer, A. Martin. Action minima among solution to a class of Euclidean scalar field equations // Commun. Math. Phys. 1978. V. 58, No. 2. P. 211-221.

9. G. Azorero, I. Alonso. Multiplicity of solutions for elliptic problems with critical exponent or with a nonsymmetric term // Trans. Amer. Math. Soc. 1991. V. 323, No. 2. P. 877-895.

10. H. Berestycki, P.L. Lions. Nonlinear scalar field equations, II. existence of infinitely many solutions // Arch. Ration. Mech. An. 1983. V. 82, No. 4. P. 347-375.

11. A. Castro, J. Cossio, J.M. Neuberger. A sign-changing solution for a superlinear Dirichlet problem. // Rocky Mt. J. Math. 1997. V. 27, No. 4. P. 1041-1053.

12. D. Costa, Z. Ding, J.M. Neuberger. A numerical investigation of sign-changing solutions to superlinear elliptic equations on symmetric domains // J. Comp. Appl. Math. 2001. V. 131, No. 1. P. 299-319.

13. T. Bartsch, T. Weth. A note on additional properties of sign changing solutions to superlinear elliptic equations // Topol. Method. Nonl. An. 2003. V. 22, No. 1. P. 1-14.

14. S.I. Pokhozhaev. On an approach to nonlinear equations // Dokl. Akad. Nauk SSSR. 1979. V. 247, No. 6. P. 1327-1331. [Sov. Math. Dokl. 1979. V. 20. P. 912-916.]

15. S.I. Pokhozhaev. On the method of fibering a solution in nonlinear boundary value problems // Trudy MIAN SSSR. 1990. V. 192. P. 146-163. [Proc. Steklov Inst. Math. 1992. V. 192. P. 157-173.]

16. Ya. Il'yasov. On a procedure of projective fibering of functionals on Banach spaces // Proc. Steklov Inst. Math. 2001. V. 232. P. 150-156.

17. Ya.Sh. Il'yasov. Non-local investigation of bifurcations of solutions of non-linear elliptic equations // Izv. RAN. Ser. matem. 2002. V. 66, No. 6. P. 19-48. [Izv. Math. 2002. V. 66, No. 6. P. 1103-1130.]

18. D. Kinderlehrer, G. Stampacchia. An Introduction to Variational Inequalities and Their Applications. Academic Press, New York. 1979.

19. M. Balabane, J. Dolbeault, H. Ounaies. Nodal solutions for a sublinear elliptic equation // Nonlinear Anal.-Theor. 2003. V. 52, No. 1. P. 219-237. 
20. N. Dunform, J.T. Shwarz. Linear Operators. P. 1. General Theory. Pure and Applied Mathematics. V. 6. New York and London, Interscience Publishers. 1958.

21. M. Willem. Minimax Theorems. Birkhauser, Boston. 1996.

22. M.N. Vrahatis. A short proof and a generalization of Miranda's existence theorem // Proc. Amer. Math. Soc. 1989. V. 107, No. 3. P. 701-703.

Vladimir Evgen'evich Bobkov, Institute of Mathematics CS USC RAS, Chernyshevskii str., 112, 450008, Ufa, Russia

E-mail: bobkovve@gmail.com 